Classification

Physics Abstracts

$68.35 \mathrm{Bs}-61.16 \mathrm{Ch}-68.55 \mathrm{Nq}$

\title{
Soft oxidation of graphite studied by XPS and STM
}

\author{
Magali Phaner-Goutorbe, Alain Sartre* and Louis Porte \\ Département de Physicochimie des Matériaux, Ecole Centrale de Lyon, BP. 163, 69131 Ecully, France
}

(Received July 4; accepted December 12, 1994)

\begin{abstract}
Résumé . - La modification de la surface de graphite après une faible oxydation dans une solution d'acide nitrique a été étudiée par spectroscopie de photoélectrons (XPS) et par microscopie tunnel (STM). Une nouvelle corrugation a été mise en évidence à l'échelle atomique par STM sur les plans de base du graphite. Elle est attribuée à la présence d'autre part démontrée par XPS, de groupements organiques créés par l'oxydation et distribués de façon régulière sur la surface. Cette oxydation douce n'altère pas les plans de base du graphite et est interprétée par la chimisorption d'atomes d'oxygène sur le graphite, les liaisons s'effectuant à partir d'électrons $\pi$ de la cellule aromatique. Les groupements organiques nécessitant des liaisons pendantes $\sigma$ pour se fixer sur la surface semblent se positionner préférentiellement sur les arètes de gros défauts physiques.
\end{abstract}

\begin{abstract}
The modification of the graphite surface after weak oxidation using a nitric acid solution has been studied by X-ray photoelectron spectroscopy (XPS) and scanning tunneling microscopy (STM). A new corrugation at the atomic scale has been revealed by STM on the basal planes. It is attributed to the presence, in other respects demonstrated by XPS, of some oxygenated groups created by oxidation and regularly distributed on the surface. This soft oxidation conserves the graphite basal planes and is interpreted by an oxygen chemisorption on graphite through a bond involving $\pi$ electrons of the aromatic cell. Organic groups which need dangling $\sigma$ bonds to fix on the surface should preferencially attach on ridges of large defects.
\end{abstract}

\section{Introduction.}

Solid carbon materials have proven to be suitable supports for metal catalysts in the chemical industry for hydrogenation reactions. As solid carbons are chemically inert, the first step of the catalyst preparation procedure usually consists in the functionalization of the carbon surface. It is well known [1-5] that some reactive sites can be obtained by an oxidation process. However this activation occurs preferentially on edge sites: steps or deep holes on the carbon surface, places with an important rate of carbon dangling bonds. So standard oxidation treatments were performed to increase the number of these "physical defects" or enhance the existing one making

* Centre de Recherches Rhône Poulenc, 52 rue de la Haie Coq, 93308 Aubervilliers Cedex, France 
them deeper and larger. Then, the distribution of the metallic precursors is essentially defined by these large defects. As these defects are randomly distributed on the carbon surface and concern finally a minority of carbon atoms, we focussed our interest in this study on the creation of a new kind of modification called here "chemical defects" splited up homogeneously at the atomic scale on the basal carbon plane. The graphite surface was chosen as carbon surface for its very low edge-to-basal site ratio. The presence of these defects was demonstrated in a previous study [6], thus in this paper we investigated the very first oxidation step of graphite to promote these anchoring atomic sites at the expense of physical defects and try to preserve the graphite basal plane flatness.

\section{Experimental.}

The oxidation treatment follows a standard procedure which consists in the immersion of freshly cleaved highly oriented pyrolitic graphite sample in a nitric acid solution (4N) at a temperature around $70-80{ }^{\circ} \mathrm{C}$. In the industrial standard procedure, the treatment is performed during several hours and creates a large number of physical defects. We only investigated small times of etching $(10-30 \mathrm{mn})$ in order to limit as much as possible these physical defects. An activation at ambient temperature was also carried out during $30 \mathrm{mn}$. Each samples were then rinced in distillated water and dried for $30 \mathrm{mn}$ at $120{ }^{\circ} \mathrm{C}$. Then they were characterized by both STM (Scanning Tunneling Microscopy) and XPS (X-ray Photoelectron Spectroscopy). STM gives informations about local electronical and topographical modifications of the surface while XPS allows a chemical identification of the species on the surface and informs also on the nature of their bondings. STM images were realized in air using either a home-build microscope or a commercial Besocke apparatus. Electrochemically etched Pt-Ir tips were employed and the images were performed in the constant current mode with currents of about $1-4 \mathrm{nA}$ and a tip bias between $\pm 0.1 \mathrm{~V}$. XPS data were obtained using a Perkin-Elmer PHI 5500 instrument operating in ultrahigh vacuum conditions with a conventional $\mathrm{AlK} \alpha$ monochromatized source.

\section{Results.}

A typical XPS survey spectrum obtained from an HOPG surface after an oxidation treatment is presented figure 1. It reveals added to the carbon core level $\mathrm{C} 1 \mathrm{~s}$ peak at around $284 \mathrm{eV}$ the presence of a new peak around $533 \mathrm{eV}$ due to the oxygen $\mathrm{O} 1 \mathrm{~s}$ orbital. This confirms that the oxidation treatment was effective even for small time of etching or low temperature. The C 1 s peak is analysed in details in figure 2 to get informations about the chemical environment of carbon atoms. Figure $2 \mathrm{~b}$ presents the typical modification obtained after oxidation. An intensive treatment was performed to present all the features which could be obtained after oxidation. In comparison with the bare graphite $\mathrm{C} 1 \mathrm{~s}$ peak shown in figure $2 \mathrm{a}$, oxidation led to a broadening of the main $\mathrm{C} 1 \mathrm{~s}$ peak and the presence of new components on the high binding energy side (Fig. $2 b)$. This disymetry of the main peak and the new features are attributed to the modification of the chemical environment of carbon atoms bonded to oxygenated groups. A deconvolution procedure gives several components labelled as following: A $(284.8 \mathrm{eV}), \mathrm{B}(285.4 \mathrm{eV}), \mathrm{C}(286.6$ $\mathrm{eV}), \mathrm{D}(288.1 \mathrm{eV}), \mathrm{E}(289.2 \mathrm{eV})$ and $\pi-\pi *(290.5 \mathrm{eV})$. Following the work of Briggs [7], the main oxygenated organic groups: single bond $\mathrm{C}-\mathrm{O}$, double bond $\mathrm{C}=\mathrm{O}$ and carboxylic group $\mathrm{O}-\mathrm{C}=\mathrm{O}$ are respectively shifted by $\approx 1.5 \mathrm{eV}, \approx 3.0 \mathrm{eV}$ and $\approx 4.5 \mathrm{eV}$ from the graphite $\mathrm{C} 1 \mathrm{~s}$ peak. These values correspond pretty well to the components $\mathrm{B}, \mathrm{C}, \mathrm{D}$. The remainding components obtained $\mathrm{A}$ and $\mathrm{E}$ can tentatively be attributed respectively to non-oxidized carbon, such as for example some alkyl 


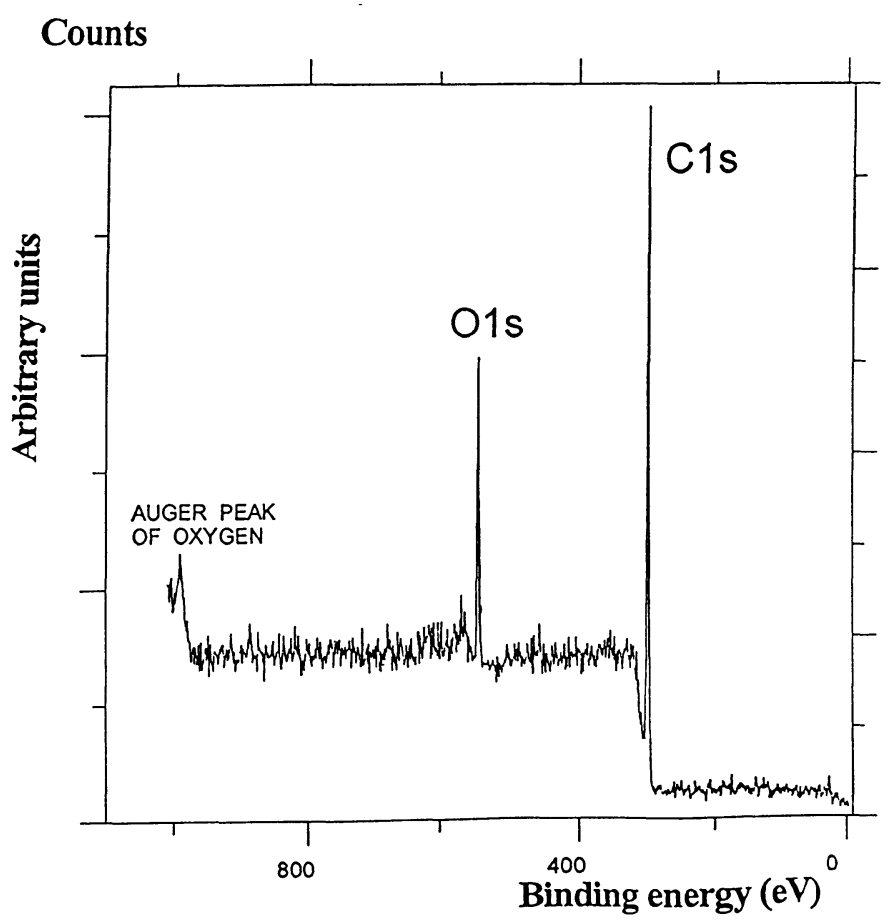

Fig. 1. - Typical XPS spectrum obtained from an HOPG surface after an oxidation treatment in $\mathrm{HNO}_{3} 4 \mathrm{~N}$ (Conditions: $10 \mathrm{mn}$ at $T=70^{\circ} \mathrm{C}$ ). An $\mathrm{O} 1 \mathrm{~s}$ peak near $533 \mathrm{eV}$ appeared in addition to the $\mathrm{C} 1 \mathrm{~s}$ peak of the graphite near $285 \mathrm{eV}$.

chain carbons $\left(-\mathrm{CH}_{2}-\right)$ and to a $\mathrm{O}_{2}-\mathrm{C}=\mathrm{O}$ group which corresponds to the stronger oxidation. The last peak at higher binding energy corresponds to the typical $\pi-\pi *$ shake up satellite. This peak present in both spectra (Figs. $2 \mathrm{a}$ and $2 \mathrm{~b}$ ) is due to electron transitions between bonding and antibonding graphite $\pi$ bands. It is clear that oxygenated organic groups were created by oxidation of the graphite surface. These components were visible on each XPS spectrum but their relative proportion changed from sample to sample depending on the preparation conditions. It seems that in the case of soft preparing conditions, the initial state of the bare graphite surface could be mainly responsible of the relative proportion of the organic components. Besides the appearance of new $C 1 \mathrm{~s}$ components, the systematic vanishing of the $\mathrm{C} 1 \mathrm{~s} \pi-\pi *$ shake up satellite was observed for all oxidized graphite samples. In the special case of figure 2 , the intensity of the $\pi-\pi *$ shake-up peak decreased by more than $60 \%$ after oxidation. As this satellite is indicative of aromaticity in graphite, it is interesting to underline that its decrease appears as a first proof of deshybridization of carbon atoms.

As the presence of organic groups was established by XPS, their localization on the surface was attempted by direct imaging with an STM. We firstly scanned large scale areas in order to find large "physical defects". The image in figure 3 illustrates a region of around $20 \mathrm{~nm} \times 20 \mathrm{~nm}$ obtained at $-0.1 \mathrm{~V}$ tip bias voltage with a tunneling current of $+1 \mathrm{nA}$. Steps and a large hole on the bottom right corner can be seen. A shiny area is observed on the edges of both step and hole. When scanning such areas the tunneling current was excited, an effect we attribute to a strong enough oxidation disturbing the surface conductivity. This confirms the known high reactivity of the physical defects i.e. basal plane edges of graphite where non statisfied carbon dangling 


\section{Counts}

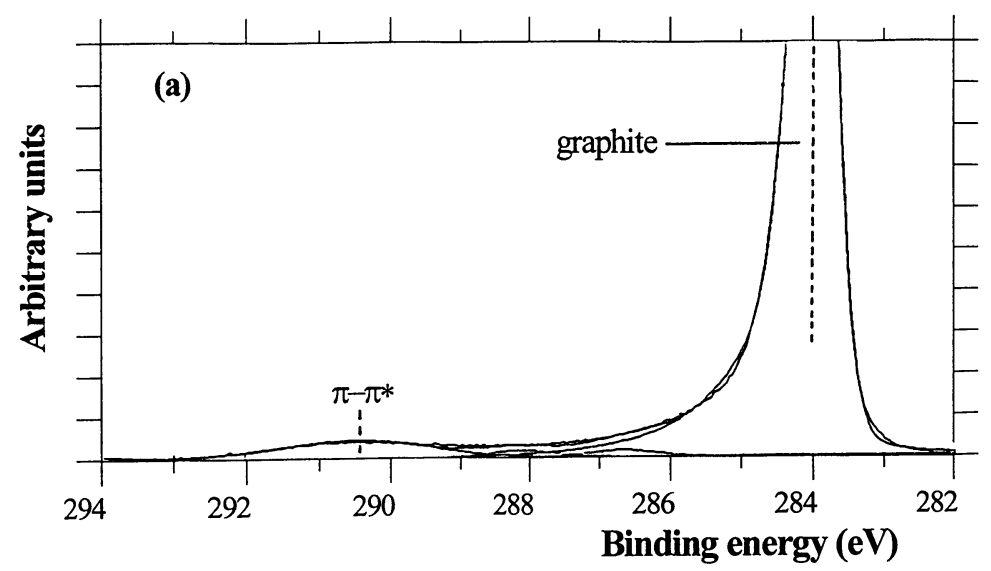

Counts

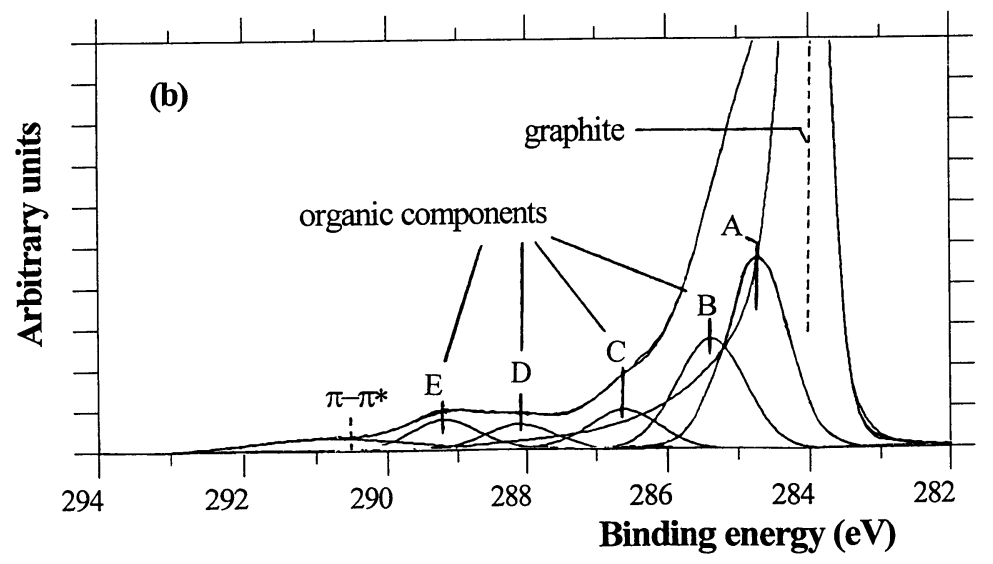

Fig. 2. - XPS C $1 \mathrm{~s}$ peaks of pure graphite sample (a) and oxidized graphite (b) after a prolongated treatment in $\mathrm{HNO}_{3} 4 \mathrm{~N}\left(T=90{ }^{\circ} \mathrm{C}\right)$. A deconvolution procedure shows new carbon components which can be assigned to different carbon functions: alkyl (A), C-O- (B), $\mathrm{C}=\mathrm{O}(\mathrm{C}), \mathrm{O}=\mathrm{C}-\mathrm{O}(\mathrm{D}),-\mathrm{O}_{2} \mathrm{C}=\mathrm{O}(\mathrm{E})$. Note also the decrease of the $\pi-\pi *$ shake up satellite.

bonds can fix various organic groups $[1,2]$. The irregular shape of the step also proves that the oxidation treatment not only created organic functions on the surface but etched the basal plane from the edge. On HOPG surface the density of edge planes is not high, thus these defects were quite scarce and difficult to find. In other words, the oxidation affects mainly the graphite surface plane and in the weak oxidation conditions the topography of the graphite basal plane is globally preserved resulting in a "smooth" oxidation.

An important modification realized by oxidation on the surface occured on the basal plane far from physical defects and was observed at atomic scale. Figure 4 gives a high resolution STM image of the graphite basal plane following weak oxidation. We can first notice that the common graphite STM image was obviously modified and a new structure appeared. One can identify the reminiscent graphite structure through the periodicity of $0.2 \mathrm{~nm}$ between rows $(0.213$ $\mathrm{nm}$ theoretically). In order to fully understand the image we drew the hexagonal structure unit 


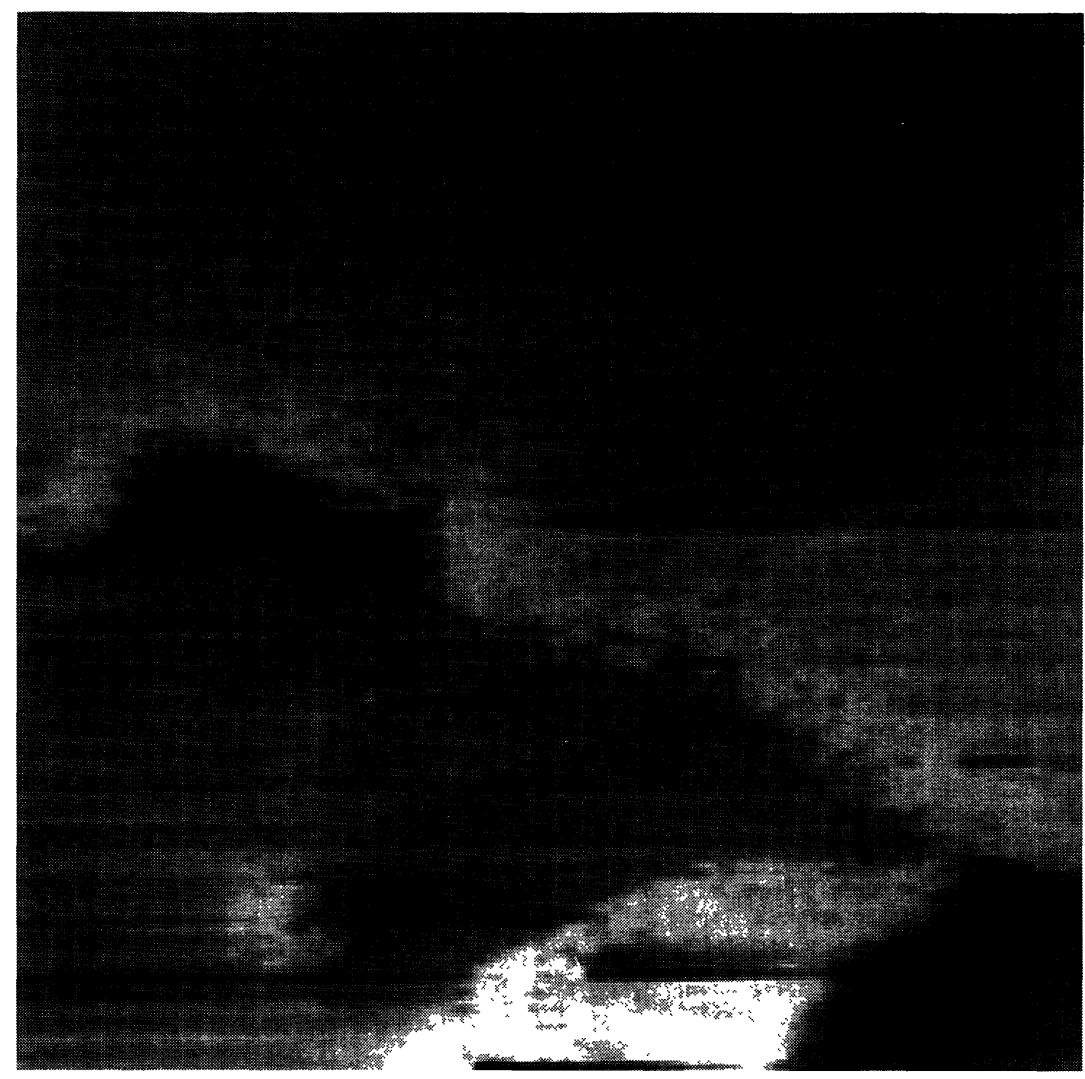

Fig. 3. - STM image of physical defects on oxidized graphite (Conditions: $20 \mathrm{mn}$ at $T=70{ }^{\circ} \mathrm{C}$ ). The oxidation effect manifests by etching along the step and shiny regions (less conductive) on ridges of steps and hole. Field of view is $20 \mathrm{~nm} \times 20 \mathrm{~nm}$. Tip bias at $-100 \mathrm{mV}$, tunnel current: $1 \mathrm{nA}$. Grey scale range $\Delta Z=7 \mathrm{~nm}$.

classically obtained in STM images of graphite. On further investigations, the $0.246 \mathrm{~nm}$ lattice parameter can be extracted nearly parallel to the $X$ scanning direction. In the basal plane of graphite two neighboring carbon atoms belong to non equivalent sites. One, the A site, gets another carbon lying directly beneath in the sublayer, another, the B site, is located above an hollow site of the sublayer. It was shown [8] that in normal experimental STM conditions, only the $\mathrm{B}$ site atoms are observed as they have larger contribution to the tunneling current than the A site atoms. Obviously, the general appearance of rows instead of well defined dots as for bare graphite indicates a modification of the electronic contribution of related $\mathrm{A}$ and $\mathrm{B}$ graphite sites. The second major difference in comparison to graphite is an alternation between white and black rows when one tries to follow a given row (Fig. 4: line (1)). Moreover this effect determines a boundary zone of weak image contrast, oriented at $30^{\circ}$ from rows (Fig. 4: line (2)). The boundary zones are regularly spaced, their separation being around $1.2 \mathrm{~nm}$ in figure 4 . The contrast modification of the rows at this boundary zone can be attributed to the presence of oxygen atoms fixed at that place and distributed homogeneously in one direction (line (2)). This kind of modification of the graphite structure was quite constantly observed on the basal plane of HOPG after the oxidation treatment even though differences were noticed on the apparent wavy structure due to 
temperature and time modifications of the oxidation treatment. This was already observed from XPS with variations in the relative intensity of the $\mathrm{C} 1 \mathrm{~s}$ components.

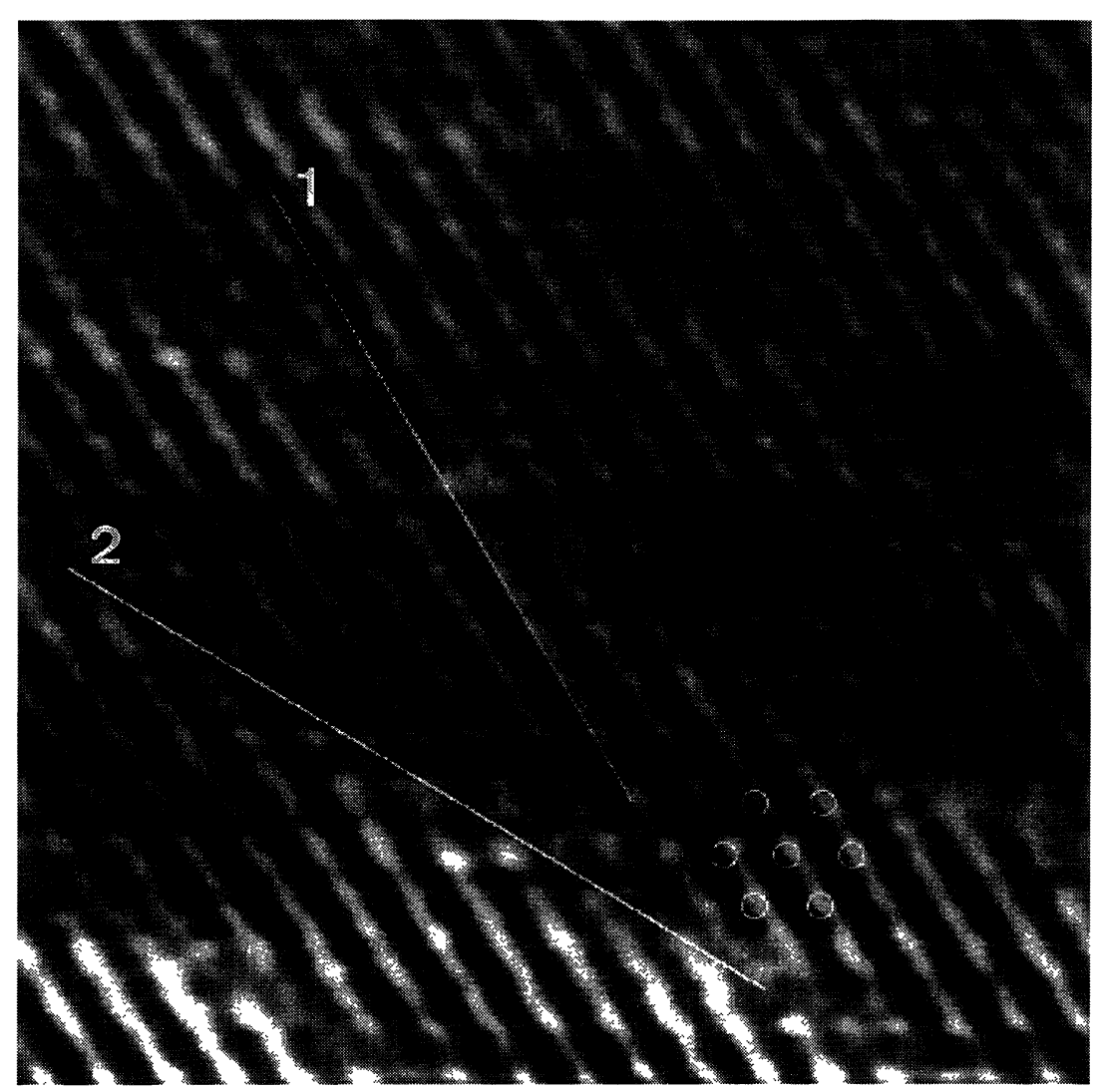

Fig. 4. - High resolution STM image on the basal plane of oxidized graphite (Conditions: $30 \mathrm{mn}$ at $T=70^{\circ} \mathrm{C}$ ). White circles represent the hexagonal graphite unit cell. Drawn lines indicate the alternation of rows (1) and the appearance of a new large corrugation (2). Field of view is $4.4 \mathrm{~nm} \times 4.4 \mathrm{~nm}$. Tip bias 30 $\mathrm{mV}$, tunneling current: $-5 \mathrm{nA}$. Grey scale range $\Delta Z=0.6 \mathrm{~nm}$.

\section{Discussion.}

The interpretation of STM images has to rely on the functionalization of the graphite surface as it was revealed by XPS. Then, the question of imaging by STM of created organic groups arises. Because the images exhibit at first sight some similarity with those obtained for organic molecules deposited on graphite [9-13], one can suggest that the oxidation creates some kind of organic layer constitued of alkyl chains terminated by organic groups. However this hypothesis is inconsistent with the separation of $0.2 \mathrm{~nm}$ found between rows while the distance between alkyl chains is around $0.45 \mathrm{~nm}$ [10]. Then we assume that because the oxidation was weak, the graphite basal plane was globally conserved but some oxygen atoms were regularly fixed on carbon atoms of 
graphite. The bonding can be effective using $\pi$ electrons [1]. This hypothesis is in agreement with the $\pi-\pi *$ satellite decrease observed by XPS, the $\sigma$ bonds are not broken and the oxidation operates through the sp2 deshybridization. Locally on the oxidized carbon some partial sp3like hybridization should take place, also inducing a distorsion of the aromatic cell. In such a description one could imagine oxygen atoms either in hydroxyl group $\mathrm{OH}$ or linked with two neighboring carbons of graphite C-O-C. Carboxylic groups which were also revealed by XPS could not in this description fix on the basal plane as they need to break $\sigma$ bonds. It is well known for stronger oxidations [1] that they are preferentially found on ridges of large defects where $\sigma$ dangling bonds are numerous. So in our experiment we suggest that carboxylic groups or even higher oxygenated component should be responsible of the oxidized areas on the edge planes.

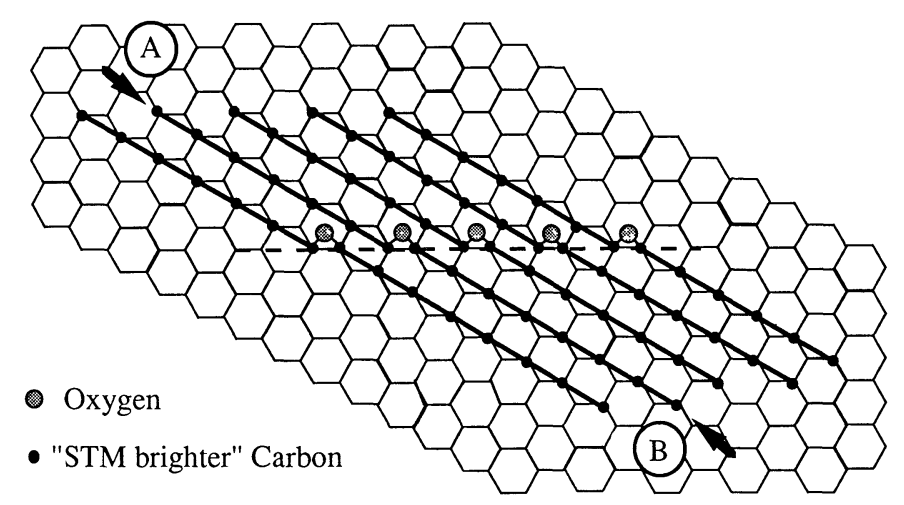

Fig. 5. - Model proposed in regards to the image in figure 4. Oxygen atoms which should chemisorb between two neighboring carbon atoms A and B (non equivalent sites) of graphite are punctual defects inducing modifications of the local density of states. Solid line $=$ white row of (1) direction of figure 4 and dashed line $=(2)$ direction of figure 4 . The adsorption of oxygen atom between A and B carbon sites is supposed to enhance the local electronic density of the graphite atoms along the privileged (1) direction. The appearance of the corrugation given by (2) line should correspond to an oxygen network.

As an illustration of this $\pi$ bonding hypothesis we propose the model presented in figure 5. One oxygen atom is bonded to two neighboring carbon atoms using the two $\pi$ electrons of the aromatic cell while $\sigma$ bonds are unaffected. As explained before, two neighbor carbon atoms are imaged differently because they belong to two non-equivalent carbon sites. In the present hypothesis, the presence of the $\mathrm{O}$ atom could change the local electronic density of states on the two carbons so that their contribution is no more the one normally observed for graphite. The chemisorption of $\mathrm{O}$ atoms can be treated as a surface defect on the surface of graphite, whose consequence is the modification of wave functions on carbon atoms neighbor to the adsorption site [14]. It is experimentally well established that defects, adsorbates, agregates, ... can modify the carbon wave functions and give various kind of electronic superstructure on graphite [15-17]. In the same way, we suggest that the row effect visible in the STM images reflects electronic changes on the carbon atoms. In this model, $\mathrm{O}$ atoms would be distributed as linear defects on graphite and should correspond to the low contrasted area of figure 4 . These $O$ atoms could modify the charge density of their neighboring carbons. In a way similar to Friedel oscillations observed near to defects, which can decay away from defects over some ten lattices constants [18], the $O$ atoms influence can extend on some nanometers. The electronic contribution of B site carbon and A site carbon localized near the oxygen atom could be modified in such a way that an electronic enhancement 
occurs along a given direction. The enhancement of a given cristallographic direction of graphite can be observed when imaging atomic defects on graphite $[14,18]$. As presented by figure 5 electronic enhancement due to the oxygen atoms should extend along a given direction and should affect both A sites and B sites situated on the two sides of the oxygen linking atom. Thus A sites on one side of oxygen would be enhanced leading to a white row on the image while B sites on the other side would also appear white. Then following a given row one goes from white to black where oxygen links A and B sites (Fig. 5). Of course to take account of the distance between low contrasted areas (1.2 nm in Fig. 4) one should finally consider that oxidation has created a large network of oxygen atoms on the graphite basal plane.

\section{Conclusion.}

In conclusion, we realized a weak oxidation of the graphite surface and demonstrated thanks to a double characterization by XPS and STM that it is possible to create functionalized sites distributed on the basal plane of graphite. It was called "smooth oxidation of graphite" because the graphite basal plane was not destroyed. STM images exhibited a new corrugation at atomic scale attributed to the presence of $\mathrm{O}$ atoms regularly bonded to $\mathrm{C}$ atoms of graphite using $\pi$ electrons. These $\mathrm{O}$ atoms were found by XPS to belong to organic groups such as $\mathrm{C}-\mathrm{OH}$ or C-O$\mathrm{C}$ created on the surface by this oxidation. XPS revealed also the presence of other oxygenated functions such as carboxylic groups which were assumed to be fixed preferentially on ridges of large defects where dangling bonds are available.

\section{Bibliographie}

[1] Carlos A., Leon y Leon D. and Ljubisa R. Radovic, in "Chemistry and Physics of Carbon", P.A. Thrower Ed., Vol. 4 (New York, Basel, Hong Kong, 1994) 213.

[2] Hubacek J.S., Brockenbrough R.T., Gammie G., Skala S.L., Lyding J.W., Latten J.L. and Shapley J.R., J. Microsc. $152(1988) 221$.

[3] Atamny F., Schlögl R., Wirth W.J. and Stephan J., Ultramicroscopy $42-44$ (1992) 660.

[4] Rabe J.P., Buchholz S. and Ritcey A.M., J. Vac. Sci. Technol. A8 (1990) 679.

[5] Porte L., Richard D. and Gallezot P., J. Microsc. 152 (1988) 512.

[6] Sartre A., Phaner M., Porte L. and Sauvion G.N., Appl. Surf. Sci. 70/71 (1993) 402.

[7] "Practical Surface Analysis by Auger and X-ray Photoelectron Spectroscopy", D. Briggs and M.P. Seah Eds., John Wiley \& Sons, LTD (Chichester, New York, Brisbane, Toronto, Singapore, 1983) 385.

[8] Batra I.P. and Ciraci S., J. Vac. Sci. Technol. A6 (1988) 313.

[9] Rabe J.P. and Buchholz S., Science 253 (1991) 424.

[10] McGonigal G.C., Bernhardt R.H. and Thomson D.J., Appl. Phys. Lett. 57 (1990) 28.

[11] Yeo Y.H., Yackoboski K., McGonigal G.C. and Thomson D.J., J. Vac. Sci. Technol. A10 (1992) 600.

[12] Rabe J.P. and Buchholz S., Phys. Rev. Lett. 66 (1991) 16.

[13] Bucher J.P., Roeder H. and Kern K., Surf. Sci. 289 (1993) 370.

[14] Mizes H.A. and Harrison W.A., J. Vac. Sci. Technol. A6 (1988) 300.

[15] Porte L., de Villeneuve C.H. and Phaner M., J. Vas. Sci. Technol. B9 (1991) 1064.

[16] Porte L., Phaner M., Noupa C., Tardy B. and Bertolini J.C., Ultramicroscopy 42-44 (1992) 1355.

[17] Xhie J., Sattler K., Muller V., Venkateswaran N. and Raina G., J. Vac. Sci. Technol. B9 (1991) 833.

[18] Mizes H.A., Foster J.S., Science 244 (1989) 559. 